\title{
Antimicrobial susceptibility of Helicobacter pylori isolates from Lower Silesia, Poland
}

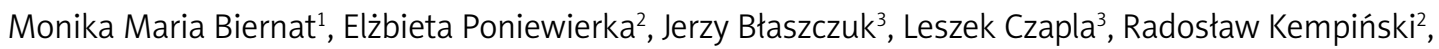

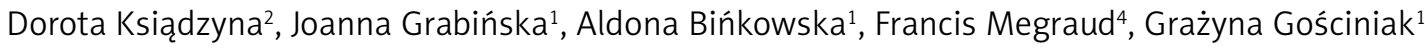

1Department of Microbiology, Medical University of Wroclaw, Poland

2Department and Clinic of Gastroenterology and Hepatology, Medical University of Wroclaw, Poland

3Department and Clinic of Gastrointestinal and General Surgery, Medical University of Wroclaw, Poland

${ }^{4}$ Laboratoire de Bactériologie, C.H.U. Pellegrin, Bordeaux, France

Submitted: 23 March 2012

Accepted: 7 November 2012

Arch Med Sci 2014; 10, 3: 505-509

DOI: 10.5114/aoms.2013.36917

Copyright (c) 2014 Termedia \& Banach

\section{Abstract}

Introduction: In recent years the failure of standard therapy for Helicobacter pylori infections has been observed, which results primarily from the increasing resistance of $H$. pylori strains to antibiotics. The aim of the study was to estimate the prevalence of antimicrobial resistance of $H$. pylori strains isolated from adult symptomatic patients with primary infection in the Lower Silesia Region in Poland.

Material and methods: One hundred and seventy-eight adults aged 19-89 years with dyspeptic symptoms suggesting gastroduodenal pathology were enrolled in the study. The study was performed in the years 2008-2011. Fifty $H$. pylori strains were isolated from gastric biopsy samples of examined patients. Antimicrobial susceptibility to 6 drugs (amoxicillin (AM), clarithromycin $(\mathrm{CH})$, metronidazole (MZ), tetracycline (TC), levofloxacin (LEV), and rifabutin (RB)) was tested by the gradient-diffusion method (E-test method).

Results: The incidence of $H$. pylori infection among examined patients was $35 \%$. From 50 isolated $\mathrm{H}$. pylori strains, $24 \%$ showed resistance to $\mathrm{CH}, 42 \%$ to $\mathrm{MZ}$ and $8 \%$ to LEV alone. Multidrug resistance was detected in $26 \%$ of strains, whereas $20 \%$ of isolates were resistant to $\mathrm{MZ}$ and $\mathrm{CH}$. Examined strains were fully susceptible to AM, TC and RB.

Conclusions: Resistance to clarithromycin strains isolated from adults of the Lower Silesia Region in Poland is high and is almost always associated with resistance to metronidazole $(\mathrm{CH}+\mathrm{MZ})$. It is necessary to continuously monitor $\mathrm{H}$. pylori resistance to drugs used in therapy, especially to clarithromycin. Verification of the existing recommendations of eradication therapy is also needed.

Key words: Helicobacter pylori, resistance, symptomatic patients.

\section{Introduction}

Resistance of bacterial strains to antibiotics has recently become a major problem in treatment of human infectious diseases. This issue also applies to treatment of Helicobacter pylori (H. pylori) infection, which remains the main cause of both duodenal and peptic ulcers and is associated with the risk of developing gastric cancer. In recent years the possible role of this infection in extragastric diseases, such as cardiovascular diseases, hema-
Corresponding author:

Monika Maria Biernat PhD

Department of Microbiology

Medical University of Wroclaw

4 Chałubińskiego St

50-368 Wroclaw, Poland

Phone: +48717841286

E-mail: mobiernat@gmail.com 
tologic diseases, eye and skin diseases, diabetes mellitus, and neurological disorders, has been postulated [1, 2]. So far antibiotics used for eradication of this bacterium include $\beta$-lactams, macrolides, tetracyclines and fluoroquinolones. Recommendations of the European Helicobacter Study Group included in the Maastricht III Consensus Report and guidelines of the Polish Society of Gastroenterology advise in first line treatment using two of three antibiotics (amoxicillin, clarithromycin or metronidazole) and a proton pump inhibitor (PPI). Use of amoxicillin with clarithromycin or metronidazole can be administered when $H$. pylori resistance to clarithromycin in the population is lower than $20 \%$ and to metronidazole is lower than $40 \%[3,4]$. The high effectiveness of the above-mentioned regimens until recently ensured eradication of $H$. pylori in most patients, but during the last few years failure of standard eradication therapy has been seen more often, due mostly to increasing resistance of Helicobacter strains to antibiotics. The factors affecting Helicobacter resistance to antibiotics are world region, age, gender and ethnic background [5]. In Poland, multicenter studies of Helicobacter strains' resistance to antibiotics were performed 10 years ago [6]. Because of difficult isolation of bacteria, the evaluation of drug sensitivity is performed most often after eradication failure. Taking into account the above issues, $H$. pylori resistance to antibiotics in the Lower Silesian Region is difficult to estimate.

The aim of the study was to evaluate the primary resistance of Helicobacter strains isolated from adults to amoxicillin, clarithromycin, metronidazole, tetracycline, levofloxacin and rifabutin.

\section{Material and methods}

The study was performed on strains isolated from adult patients of the Lower Silesia Region in Poland in the years 2008-2011. The study was part of a multicenter project on surveillance of $H$. pylori resistance in different European countries conducted in the years 2008-2010 [7]. Our study involved 178 patients who underwent endoscopic examination of the upper gastrointestinal tract due to complaints from the upper gastrointestinal tract, such as nausea, vomiting, or abdominal pain, suggesting the presence of pathology. Patients had not been diagnosed and treated for $H$. pylori infection. Patients who had previously had $H$. pylori infection or received antibiotics within the last 2 months were excluded. Other exclusion criteria were parasitic diseases, allergies and autoimmune diseases. Informed written consent was obtained from each patient. The study was approved by the Bioethics Committee of Wroclaw Medical University, Approval No. 226/2011. The patients had the following conditions: duodenal ulcer $(n=7)$, chronic gastritis $(n=114)$, chronic gastritis and duodenitis $(n=18)$, gastroesophageal reflux disease (GERD) $(n=15)$, and other diseases $(n=24)$, including hiatal hernia and polyps. Biopsies from the antrum and, in the case of present changes, from the corpus were taken from each patient during endoscopy of the upper gastrointestinal tract for histopathology and microbiology. Biopsies collected for microbiological examination were placed immediately after collection in sterile saline $(0.15 \mathrm{M} \mathrm{NaCl})$ and processed within 2 to $3 \mathrm{~h}$ in a microbiological laboratory. The gastric biopsies were examined by direct Gram stain and placed on the following media: Columbia agar medium (Difco) with horse blood (7\%) and selective supplement (Dent), containing vancomycin $10 \mathrm{mg} / \mathrm{l}$, trimethoprim $10 \mathrm{mg} / \mathrm{l}$, cefsulodin $5 \mathrm{mg} / \mathrm{l}$ and amphotericin B $5 \mathrm{mg} / \mathrm{l}$. The cultures were incubated for 3 days at $37^{\circ} \mathrm{C}$ under microaerophilic conditions. The strains were identified as $H$. pylori by Gram stain morphology, positive culture and positive catalase, oxidase and urease tests. After the primary isolation and identification, the strains were kept frozen at $-70^{\circ} \mathrm{C}$ in Brucella broth containing $15 \%$ glycerol. Then the drug sensitivity was determined by gradient diffusion (E-test, BioMerieux) to six antibiotics: amoxicillin (AM), clarithromycin (CH), metronidazole $(M Z)$, tetracycline $(T C)$, levofloxacin (LEV) and rifabutin (RB) by the method described by Glupczynski et al. [8]. Microorganisms obtained during the 72-hour culture were suspended in brainheart infusion broth $(\mathrm{BHI})$ with a density of 3 on the McFarland scale, $10^{8}$ cells (CFU/ml). In the next stages the bacterial suspension was inoculated on Mueller-Hinton medium (Becton Dickinson) supplemented with $10 \%$ horse blood. Then E-test antibiotic impregnated with a gradient of concentration was imposed, to determine the value of the minimum inhibitory concentration (MIC) for the growth of bacteria. Incubation was carried out for $72 \mathrm{~h}$ at $37^{\circ} \mathrm{C}$ in a microaerophilic atmosphere. At the end of incubation, the reading was based on the size of the zone of inhibition of $H$. pylori bacilli in the form of an ellipse. Criteria for interpretation of results: MIC $(\mu \mathrm{g} / \mathrm{ml})$ for resistant strains: amoxicillin $>0.5$, clarithromycin $>1$, metronidazole $>8$, tetracycline $>1$, levofloxacin $>0.5$ and rifabutin $>1$ $[8,9]$.

\section{Statistical analysis}

Statistical analysis was performed by $\chi^{2}$ test with or without Yates' correction and $\chi^{2}$ test among age groups. A $p$ value $<0.05$ was considered significant for all tests.

\section{Results}

A total of 178 patients aged $18-89$ years were included. The patients' age, gender and clinical diag- 
nosis are presented in Table I. Among all patients, H. pylori infection was found in 62 (34.83\%) individuals. Helicobacter pylori was isolated in 50 cases and the antibiotic sensitivity was determined. In 3 cases the culture was not successful despite positive direct Gram stain. In another 9 cases evaluation of drug sensitivity was impossible despite positive culture. Among 50 evaluated isolated H. pylori strains 12 (24\%) showed resistance to clarithromycin, $21(42 \%)$ to metronidazole and 4 (8\%) to levofloxacin (Table II). Resistance to clarithromycin and metronidazole was found more often than to amoxicillin, tetracycline, levofloxacin and rifabutin $(p<0.05)$. Different sensitivity to clarithromycin and metronidazole, depending on the age of the patient, has been demonstrated (Table III). The highest clarithromycin resistance (30\%) was found in young individuals, whereas the resistance to metronidazole increased with age. The multidrug resistant strains (for two or more antibiotics) were most often isolated from the youngest patients or for patients above 65 years of age, though the differences were not statistically significant $(p>0.05)$. Multidrug resistant strains were isolated from thirteen patients, mostly women with gastritis (Table IV). Among these 13 (26\%) strains, 10 (20\%) were resistant to clarithromycin and metronidazole, $2(4 \%)$ to metronidazole and levofloxacin, and $1(2 \%)$ strain was resistant to 3 antibiotics: clarithromycin, metronidazole and levofloxacin. Most metronidazole resistant isolates were found in a low drug concentration $(256 \mu \mathrm{g} / \mathrm{ml})$.
Table I. Characteristics of examined patients

\begin{tabular}{|lcc|}
\hline Parameter & Number & Percentage \\
\hline Adults & 178 & 100 \\
\hline Sex & & \\
\hline Momen & 110 & 62 \\
\hline Gender [years] & 68 & 38 \\
\hline $19-44$ & & \\
\hline $45-64$ & 61 & 34 \\
\hline$>65$ & 69 & 39 \\
\hline Clinical diagnosis & 48 & 27 \\
\hline Duodenal ulcer & 7 & 4 \\
\hline Chronic gastritis & 114 & 64 \\
\hline Chronic gastritis and duodenitis & 18 & 10 \\
\hline GERD & 15 & 8 \\
\hline Other diseases* & 24 & 14 \\
\hline
\end{tabular}

*Hiatal hernia, polyps

\section{Discussion}

Knowledge of local antibiotic sensitivity is one of the most important keys to therapy success. Comparing the results of our study to $\mathrm{H}$. pylori drug resistance evaluated 10 years ago in Poland and the Lower Silesia Region, we found a considerable increase of $H$. pylori resistance to clarithromycin and metronidazole $[6,10]$. In our region,

Table II. The primary resistance and MIC values for 50 isolated strains

\begin{tabular}{|c|c|c|c|c|c|}
\hline \multirow[t]{2}{*}{ Antibiotic } & \multicolumn{4}{|c|}{ Resistant strains } & \multirow[t]{2}{*}{ Range } \\
\hline & Number & $\%$ (95\% confidence interval) & $M / C_{50}$ & $M / C_{90}$ & \\
\hline $\mathrm{CH}$ & 12 & $24(16.9-31.0)$ & 0.023 & 32 & $0.016-256$ \\
\hline$M Z$ & 21 & $42(25.3-58.7)$ & 256 & 256 & $0.016-256$ \\
\hline LEV & 4 & $8(4.9-15.0)$ & 0.125 & 3 & $0.002-32$ \\
\hline AM & 0 & $0(0.0-0.0)$ & 0.016 & 0.08 & $0.016-256$ \\
\hline $\mathrm{RB}$ & 0 & $0(0.0-0.0)$ & 0.002 & 0.02 & $0.002-32$ \\
\hline TC & 0 & $0(0.0-0.01)$ & 0.047 & 0.125 & $0.016-256$ \\
\hline
\end{tabular}

CH - clarithromycin, MZ - metronidazole, LEV - levofloxacin, AM - amoxicillin, RB - rifabutin, TC - tetracycline, MIC 50 - minimal inhibitory concentration required to inhibit the growth of $50 \%$ of organisms, $M I C_{90}$ - minimal inhibitory concentration required to inhibit the growth of $90 \%$ of organisms

Table III. Distribution of primary resistance of examined H. pylori strains in different age groups

\begin{tabular}{|c|c|c|c|c|c|c|c|c|c|c|c|c|c|}
\hline \multirow{3}{*}{$\begin{array}{l}\text { Age } \\
\text { group }\end{array}$} & \multirow{3}{*}{$\begin{array}{l}\text { Number } \\
\text { of } H . \text { pylori } \\
\text { strains }\end{array}$} & \multicolumn{6}{|c|}{ Isolates with resistance } & \multicolumn{6}{|c|}{ Multidrug resistant isolates } \\
\hline & & \multicolumn{2}{|c|}{$\mathrm{CH}$} & \multicolumn{2}{|c|}{$M Z$} & \multicolumn{2}{|c|}{ LEV } & \multicolumn{2}{|c|}{$\mathrm{MZ}+\mathrm{CH}$} & \multicolumn{2}{|c|}{$\mathrm{MZ}+\mathrm{CH}+\mathrm{LEV}$} & \multicolumn{2}{|c|}{$M Z+L E V$} \\
\hline & & $n$ & $\%$ & $n$ & $\%$ & $n$ & $\%$ & $n$ & $\%$ & $n$ & $\%$ & $n$ & $\%$ \\
\hline $19-44$ & 20 & 6 & 30 & 5 & 25 & 2 & 10 & 4 & 20 & 1 & 5 & 1 & 5 \\
\hline $45-64$ & 19 & 3 & 16 & 11 & 58 & 1 & 5 & 3 & 16 & 0 & 0 & 1 & 5 \\
\hline$>65$ & 11 & 3 & 27 & 5 & 45 & 1 & 9 & 3 & 27 & 0 & 0 & 0 & 0 \\
\hline Total & 50 & 12 & 24 & 21 & 42 & 4 & 8 & 10 & 20 & 1 & 2 & 2 & 4 \\
\hline
\end{tabular}


Table IV. Group of patients from whom multi-resistant H. pylori strains were isolated

\begin{tabular}{|lccccccccc|}
\hline No. & Age & Gender & Diagnosis & \multicolumn{7}{c|}{ MIC $[\mu \mathrm{g} / \mathrm{ml}]$} \\
\cline { 5 - 9 } & & & & CH & MZ & AM & TC & LEV & RB \\
\hline 1 & 25 & F & Gastritis & 8 & $>256$ & 0.047 & $<0.016$ & 32 & $<0.002$ \\
\hline 2 & 25 & F & Gastritis & 6 & $>256$ & 0.016 & 0.094 & 0.125 & $<0.002$ \\
\hline 3 & 25 & F & Gastritis & 32 & 256 & 0.008 & $<0.016$ & 0.125 & 0.002 \\
\hline 4 & 27 & F & Gastritis & $<0.016$ & $>256$ & 0.016 & 0.047 & $>256$ & $<0.002$ \\
\hline 5 & 28 & M & Gastritis & 32 & $>256$ & 0.023 & 0.125 & 0.125 & $<0.002$ \\
\hline 6 & 32 & F & Gastritis & 4 & 256 & $<0.002$ & $<0.016$ & 0.032 & $<0.002$ \\
\hline 7 & 53 & M & Gastritis & 256 & 256 & 0.002 & 0.047 & 0.064 & $<0.002$ \\
\hline 8 & 55 & F & Gastritis & 3 & 8 & 0.006 & 0.016 & 0.032 & $<0.002$ \\
\hline 9 & 57 & F & Gastritis & 0.023 & 128 & 0.016 & 0.047 & 3 & $<0.002$ \\
\hline 10 & 60 & F & Gastritis, hernia & 12 & $>256$ & 0.016 & 0.032 & 0.064 & $<0.002$ \\
\hline 11 & 68 & F & Gastritis & 32 & $>256$ & 0.064 & 0.047 & 0.125 & $<0.002$ \\
\hline 12 & 74 & M & Gastritis & 6 & 256 & 0.016 & 0.047 & 0.125 & $<0.002$ \\
\hline 13 & 76 & M & Gastritis, hernia & 8 & $>256$ & 0.016 & 0.064 & 0.19 & $<0.002$ \\
\hline
\end{tabular}

F-female, $M-$ male

resistance to clarithromycin in adults is high $(24 \%$ formerly $15 \%$ ). A stable increasing trend of $\mathrm{H}$. pylori resistance to clarithromycin is recently being observed; globally $5-28 \%$ of $H$. pylori strains are clarithromycin resistant. Based on multicenter studies performed in Europe, notable differences in clarithromycin resistance were found among different European countries (5-20\%) [11-13]. In our study, we found metronidazole resistance in up to $42 \%$ of patients deriving from our region, compared to $27-52 \%$ formerly $[6,14]$. Resistance of $\mathrm{H}$. pylori to metronidazole varies from $20 \%$ to $80 \%$, for instance, in Japan 1-12\%, USA $20-40 \%$, Europe $50-80 \%$, Mexico $70-80 \%$, Africa $80 \%$ [1113]. Up to now in Poland there have been no amoxicillin or tetracycline resistant strains found, as was also confirmed in our study [6]. In other countries, resistance to these two antibiotics is constantly increasing: for amoxicillin in Bulgaria $2.4 \%$, Spain 1.4\%; and for tetracycline in Sweden $0.3 \%$, Great Britain 0.5\%, Korea 8.8\% [13, 15, 16]. The analysis performed in several European centers proved high efficacy of levofloxacin, one of the new fluoroquinolones, in combination with amoxicillin and a proton pump inhibitor for Helicobacter eradication therapy. This treatment could be a good alternative for standard therapy failure [17-19]. Fast growth of resistance to fluoroquinolones used commonly for other indications should also be considered. In our study we observed slight $H$. pylori resistance to levofloxacin, though it was not previously used in the eradication therapy. Rifabutin could be another possible alternative for unsuccessful therapy, but it can be used after several treatment failures [20].
In our study $20 \%$ of $\mathrm{H}$. pylori isolates were resistant to metronidazole and clarithromycin together. The double resistance to these antibiotics in Europe is low (0.6-8.9\%), while in Asia the level is $8.3 \%$ [11, 13]. Common use of clarithromycin in upper respiratory tract infections and metronidazole in gynecological and parasitic infections can considerably increase the $H$. pylori resistance to these two agents [5]. This theory can explain the high incidence of clarithromycin-resistant $H$. pylori strains and multi-drug resistance in young adults, especially in women. In Poland antibiotic abuse, especially macrolides in upper respiratory tract infections, is common [21]. Some authors report that Helicobacter eradication failure is not only caused by antibiotic resistance but also may depend on other bacterial or host factors [22].

The coincidence of resistance to levofloxacin with clarithromycin and/or metronidazole resistance might also be distressing. It seems that the frequency of such multidrug resistant Helicobacter isolates will increase in the future.

This study has some limitations. The analysis included only 50 strains. It is necessary to conduct studies on a larger number of $H$. pylori strains from different regions of the country, so as to know the exact scale of the problem of primary resistance. Furthermore, the strains derived from patients with varying clinical diagnosis, especially with chronic gastritis. In the group studied, there was a small percentage of patients with peptic ulcer disease, in which the profile of phenotypic susceptibility of $H$. pylori strains may vary. In conclusion, we found in our study that resistance of $H$. pylori isolates from adults in the Lower Silesia Region to clarithromycin 
is high and almost always connected with resistance to metronidazole $(\mathrm{MZ}+\mathrm{CH})$. Primary resistance to levofloxacin is low, suggesting a possible alternative in case of $\mathrm{H}$. pylori eradication failure. Constant monitoring of $H$. pylori resistance to antibiotics used in the therapy is necessary in our region, especially for clarithromycin, as well as verification of guidelines for Helicobacter eradication.

\section{References}

1. Budzyński J. The favourable effect of Helicobacter pylori eradication therapy in patients with recurrent angina-like chest pain and non-responsive to proton pump inhibitors - a preliminary study. Arch Med Sci 2011; 7: 73-80.

2. Hosseinzadeh M, Khosravi A, Saki K, Ranjbar R. Evaluation of Helicobacter pylori infection in patients with common migraine headache. Arch Med Sci 2011; 7: 844-9.

3. Malfertheiner P, Megraud F, O'Morain C, et al. and the European Helicobacter Study Group (EHSG). Current concepts in the management of Helicobacter pylori infection: the Maastricht III Consensus Report. Gut 2007; 56: 772-81.

4. Dzieniszewski J, Jarosz M; the Polish Society of Gastroenterology Helicobacter pylori Working Group for the Helicobacter pylori infection: Consensus of the Polish Society of Gastroenterology Helicobacter pylori Working Group on diagnostic and therapeutic approaches for the Helicobacter pylori infection (2008). Gastroenterol Pol 2008; 5: 323-31.

5. Megraud F. H pylori antibiotic resistance: prevalence, importance, and advances in testing. Gut 2004; 53: 1374-84.

6. Łaszewicz WW. H. pylori infection in Poland- epidemiological studies in children and adults, including the risk of stomach and duodenal ulcer disease and gastric cancer. Academia Medica Bialostocensis 2003; 3-39.

7. Megraud F, Kist M, Lopez-Brea M, et al. Surveillance of Helicobacter pylori resistance to antibiotics in Europe 2008-2009. Gastroenterology 2011; 140 (Suppl 1): 1715.

8. Glupczynski Y, Broutet N, Cantagrel A, et al. Comparison of the E-test and agar dilution method for antimicriobial susceptibility testing of Helicobacter pylori. Eur J Clin Microbiol Infect Dis 2002; 21: 549-52.

9. Megraud F, Lehours P. Helicobacter pylori detection and antimicrobial susceptibility testing. Clin Microbiol Rev 2007; 20: 280-322.

10. Matysiak-Budnik T, Knapik Z, Megraud F, et al. Helicobacter pylori infection in Eastern Europe: seroprevalence in the Polish population of Lower Silesia. Am J Gastroenterol 1996; 91: 12-4.

11. Megraud F. Helicobacter pylori and antibiotic resistance. Gut 2007; 56: 1502.

12. Selgrad M, Malfertheiner P. Treatment of Helicobacter pylori. Curr Opin Gastroenterol 2011; 27: 565-70.

13. De Francesco V, Giorgio F, Hassan C, et al. Worldwide H. pylori antibiotic resistance: a systemic review. J Gastrointestin Liver Dis 2010; 4: 409-14.

14. Boyanova L, Mentis A, Rozynek E, et al. The status of antimicrobial resistance of $\mathrm{H}$. pylori in Eastern Europe. Clin Microbiol Infect 2002; 7: 388-96.

15. Boyanova L. Prevalence of multidrug-resistant Helicobacter pylori in Bulgaria. J Med Microbiol 2009; 58: 930-5.

16. Cuadrado-Lavin A, Salcines-Caviedes JR, Carrascosa MF, et al. Antimicrobial susceptibility of Helicobacter pylori to six antibiotics currently used in Spain. J Antimicrob Chemother 2012; 67: 170-3.

17. Egan BJ, Marzio L, O' Connor H, O'Morain C. Treatment of Helicobacter pylori infection. Helicobacter 2008; 13 (Suppl. 1): 35-40.

18. Liou JM, Lin JT, Chang CY, et al. Levofloxacin-based and clarithromycin-based triple therapies as first-line and second-line treatments for Helicobacter pylori infection: a randomised comparative trial with crossover design. Gut 2010; 59: 572-8.

19. Romano M, Cuomo A, Gravina AG, et al. Empirical levofloxacin-containing versus clarithromycin-containing sequential therapy for Helicobacter pylori eradication: a randomised trial. Gut 2010; 59: 1465-70.

20. Gisbert JP, Calvet X. Rifabutin in the treatment of refractory Helicobacter pylori infection. Aliment Pharmacol Ther 2012; 35: 209-21.

21. Elseviers MM, Ferech M, Vander Stichele RH, Goossens H; ESAC project group. Antibiotic use in ambulatory care in Europe (ESAC data 1997-2002): trends, regional differences and seasonal fluctuations. Pharmacoepidemiol Drug Saf 2007; 16: 115-23.

22. Graham DY, Shiotani A. New concepts of resistance in the treatment of Helicobacter pylori infections. Nat Clin Pract Gastroenterol Hepatol 2008; 6: 321-31. 